\title{
Inversión socialmente responsable Socially responsible investment
}

\author{
Alberto Muñoz Fernández \\ Universidad de Navarra \\ amunfer@unav.es
}

Recibido / received: 21/07/2016

Aceptado / accepted: 14/09/2016

DOI: $\underline{\text { http://dx.doi.org/10.20318/eunomia.2016.3293 }}$

\section{Resumen}

La inversión socialmente responsable supone integrar consideraciones sociales, ambientales o éticas en el proceso de inversión, sin renunciar a la búsqueda de ganancias. Las estrategias que se pueden seguir para incorporar estos elementos son variadas. Históricamente la ISR ha pasado de verse como una cuestión meramente ética, a considerarse que, a largo plazo, el tener en cuenta los riesgos sociales, ambientales, éticos o de buen gobierno es beneficioso para obtener retornos de un modo más sostenible. No es de extrañar que hayan surgido iniciativas desde instancias internacionales, del ámbito bursátil o de los propios inversores para fomentar estas prácticas. También son cada vez más frecuentes las leyes encaminadas a facilitar la ISR.

\section{Palabras clave}

Inversión socialmente responsable; inversión ética; sostenibilidad; principios de Ecuador; principios para la inversión responsable; transparencia; aspectos no financieros.

\begin{abstract}
Socially responsible investing involves integrating social, environmental or ethical considerations in the investment process without giving up the pursuit of profit. The strategies that can be followed to incorporate these elements are varied. Historically, SRI has evolved from an ethical conduct, to consider that in the long term, taking into account the social, environmental, ethical or governance risks is beneficial for returns on a more sustainable way. It is not surprising that initiatives have arisen from international bodies, the securities sector or investors themselves to promote these practices. Moreover, laws to encourage responsible investment are becoming more frequent.
\end{abstract}

\section{Keywords}

Socially Responsible Investment; ethical investment; sustainability; Equator Principles; principles for responsible investment; transparency; non financial issues.

SUMARIO. 1. Concepto y terminología relacionada. 2. Origen y evolución de la ISR. 3. ¿Cómo se lleva a la práctica? 4. Iniciativas para el fomento de la inversión responsable. 4.1 Introducción. 4.2. Iniciativas en el ámbito bursátil. 4.3. Iniciativas internacionales. 4.3.1. Introducción. 4.3.2. Principios para la Inversión Responsable (PRI Initiative). 4.3.3. Instituciones destinadas específicamente a orientar a las entidades financieras. 4.3.4. El inversor como supervisor y garante

\footnotetext{
*Este trabajo se enmarca en la EU Action Grant "Business and Human Rights Challenges for CrossBorder Litigation in the EU”, 2014- 2016.
} 
del respeto del medioambiente y cuestiones sociales en la realización de grandes proyectos: los Principios de Ecuador. 4.5. Legislación europea y nacional. 5. Críticas a la inversión responsable y valoración final.

\section{Concepto y terminología relacionada}

Inversión socialmente responsable (ISR) es aquella que asume de modo explícito la relevancia de factores que van más allá de los meramente económicos, como el cuidado del medioambiente, el impacto social, el buen gobierno o aspectos éticos; tiene en cuenta la importancia del buen funcionamiento y la estabilidad del mercado en su conjunto a largo plazo; y es consciente de que la generación de beneficios a largo plazo y de modo sostenible depende de sistemas sociales, medioambientales y económicos que funcionen bien y de modo estable (PRI: 1).

Es importante destacar que la intención de obtener retornos es también un elemento esencial de la ISR, aunque se tomen en cuenta, además, otros factores. En este sentido, los inversores responsables no se diferencian de otros inversores. Por esta razón, algunos autores consideran que las actividades de inversión que por su finalidad social aceptan a priori unos retornos inferiores a los del mercado no entran en el concepto de ISR (Sparkes, 2001: 195; Ransome y Sampford, 2016: 11). Lo distintivo de la ISR es que permite la confluencia de los intereses de la sociedad y los del inversor (PRI: 3).

Pese a que algunos autores distinguen la "inversión ética" de la ISR, comprendiendo la primera solamente aquella que se basa en exigir que el destinatario cumpla con los valores de una organización (Sparkes, 2001: 199) ${ }^{1}$, parece más adecuado optar por un concepto de ISR que comprendería cualquier inversión que tenga en cuenta aspectos distintos de los meramente económicos. Por eso consideraremos como equivalentes los conceptos ISR, inversión ética o inversión responsable (Ransome y Sampford, 2016: 10).

Cabe considerar, por lo tanto, que la ISR es aquella que busca resultados financieros, pero teniendo al mismo tiempo en cuenta otros criterios. Refleja, en definitiva, una preocupación del inversor por cómo se generan sus beneficios.

Algunos autores optan por definirla como un proceso. Se definiría entonces como "un proceso de inversión que considera las consecuencias sociales medioambientales, tanto positivas como negativas, de inversión en el contexto de un análisis financiero riguroso" (Olcese, Rodríguez y Alfaro, 2008: 224).

Es un término ligado al de la responsabilidad corporativa, que en el Libro Verde de la Comisión Europea de 2001 se definió como "la integración voluntaria, por parte de las empresas, de las preocupaciones sociales y medioambientales en sus operaciones comerciales y sus relaciones con sus interlocutores" (Comisión Europea, 2001); y posteriormente como "la responsabilidad de las empresas por sus impactos sobre la sociedad" (Comisión Europea, 2011). Podríamos decir que la práctica de la inversión responsable debe mover a las empresas a actuar de este modo, o al menos esa es su finalidad ${ }^{2}$. De alguna manera, los inversores responsables están indicando a las empresas en las que invierten qué responsabilidades sociales debe asumir, más allá de los resultados financieros que

\footnotetext{
${ }^{1}$ En opinión de este autor, en el caso de la "inversión ética" la motivación básica de la organización no debe ser el dinero. Sin embargo, coincidimos con Ransome y Sampford en que toda inversión busca obtener rendimientos, con independencia de que estos se dediquen después a fines altruistas, por lo que no conviene establecer esta distinción (Ransome y Sampford, 2016: 10).

${ }^{2}$ Esta cuestión ha sido estudiada con detenimiento por Zarbafi, E. M. (2011).
} 
les reporten (Ransome y Sampford, 2016: 12; Zerk, 2006: 24-25). Para cerrar este círculo virtuoso sería necesario que el consumidor actuara también de modo responsable. De esta forma el objetivo de alcanzar un desarrollo más sostenible sería un hecho, tanto para las empresas como de su entorno ${ }^{3}$ : "la contribución de la empresa a la sostenibilidad del entorno en el que compite no sólo no menoscaba sino que, aunque no garantiza, sí que contribuye a la propia sostenibilidad de la empresa" (Olcese, Rodríguez y Alfaro, 2008: 15). Todos los datos señalan la creciente importancia de las empresas en la sociedad y en la economía: cuanto mayor es el poder, mayor es la responsabilidad. En este sentido, la responsabilidad social se debe ver como una oportunidad de cambio. Como ha señalado el Secretario General de las Naciones Unidas, "la sostenibilidad es una necesidad a escala global (...) y Naciones Unidas cree que los inversores tienen un papel esencial para lograrlo" (Ban Ki-moon, 2016).

\section{Origen y evolución de la ISR}

La mayor parte de la doctrina coincide en señalar como primer hito de la ISR el rechazo de ciertos movimientos religiosos a la financiación de empresas cuya actividad consideraban inmoral, como por ejemplo, el juego, la venta de alcohol o armas. Se considera como antecedente más remoto las prácticas de los cuáqueros que, ya en el siglo XVIII, excluían de sus relaciones económicas a las empresas implicadas en el tráfico de esclavos (Molthan, 2003: XII; Olcese, Rodríguez y Alfaro, 2008: 225).

No obstante, se considera que el movimiento de la ISR nace en 1970, con las primeras resoluciones de accionistas contra empresas que invertían o que trabajaban bajo el régimen del apartheid en Sudáfrica. Después fueron los propios gobiernos los que establecieron prohibiciones de invertir en este país. Otro hito importante fue la creación en 1971 de un fondo especial para aquellos inversores que no querían obtener beneficios que se derivasen de la Guerra del Vietnam ${ }^{4}$. De las mismas fechas son los primeros estudios que demostraron cómo de las inversiones que no son socialmente responsables se derivan a menudo malos resultados financieros (Molthan, 2003: XIII).

En los años 80 el movimiento se fue institucionalizando ante la creciente concienciación que contribuyeron a crear los grupos pacifistas, ecologistas y proderechos civiles. Para unificar a estos activistas se crearon, entre otros, el American Social Investment Forum o el UK Social Investment Forum (Molthan, 2003: XIII). En los años 90, la aparición de los índices bursátiles a los que haremos referencia, supuso el impulso definitivo de esta tendencia ${ }^{5}$.

Hoy existen numerosas iniciativas privadas, nacionales e internacionales que tratan de fomentar la toma en consideración de factores como el medioambiente, el impacto social y el buen gobierno.

Se puede concluir que si en un primer momento la IR llevaba a "castigar" a las empresas transgresoras (negative screening o divestment), en los últimos tiempos ha ido ganando fuerza la parte positiva de este movimiento, que lleva a

\footnotetext{
${ }^{3}$ Es la idea que subyace en la Comunicación de la Comisión de las Comunidades Europeas relativa a la responsabilidad social de las empresas: una contribución empresarial al desarrollo sostenible (2002). Como se ha señalado, la empresa responsable y sostenible debe superar planteamientos tácticos e instrumentales, e "integrar la RC [Responsabilidad Corporativa] en los valores, gobierno, estrategia y operaciones" (Olcese, Rodríguez y Alfaro, 2008: 15).

${ }^{4}$ El Pax World Club fue creado por un grupo de Metodistas.

${ }^{5}$ Sobre los orígenes de la ISR, véase también Ransome y Sampford (2016: 14-20).
} 
apostar por las empresas que se comportan de forma responsable (Olcese, Rodríguez y Alfaro, 2008: 226; Molthan, 2003: XV).

3. ¿Cómo se lleva a la práctica?

Existen diferentes estrategias para llevar a cabo una inversión responsable. Algunos expertos señalan el screening o selección, y el activismo accionarial, como las tácticas más frecuentes (Olcese, Rodríguez y Alfaro, 2008: 224). Mientras que el primero se centra en el momento de decidir en qué empresas o proyectos invertir, el segundo se refiere a las posibilidades de influencia que se derivan de ser titular de acciones. En otras palabras, se puede optar por premiar o castigar a las empresas en función de su actuación responsable o irresponsable, o tratar de mejorar el comportamiento de la empresa "desde dentro".

(a) Dentro de la primera fórmula, el inversor tiene en cuenta dos tipos de criterios: negativos -evitando invertir en empresas, sectores o países que siguen malas prácticas medioambientales, sociales o de gobernanza (ESG)- y positivos que inclinan a invertir en empresas y proyectos responsables- (Olcese, Rodríguez y Alfaro, 2008: 224).

Teniendo en cuenta los elementos positivos y negativos se adopta la decisión. En función de cómo se ponderen estos factores, los autores mencionados han distinguido varios criterios a la hora de elegir el destino de la inversión: 1) todo o nada, que lleva a descartar a las empresas que incumplan un criterio de responsabilidad de los que emplea el inversor; 2) impacto proporcional, ante la dificultad de encontrar empresas intachables, se opta por ponderar lo positivo y lo negativo; 3) impacto directo o secundario, descartando sólo a las que causan daños directos; 4) mejor de la industria, eligiendo a la "más responsable" del sector (incentiva a las empresas a ser las mejores en su ámbito y permite al inversor introducirse en sectores sensibles); 5) impacto potencial, cuando además de las reales, se toman en consideración las transgresiones potenciales (Olcese, Rodríguez y Alfaro, 2008: 224-225; Molthan, 2003: XV-XIX).

Cabe, por tanto, la opción de invertir en empresas responsables o dejar de invertir en empresas no responsables. La opción positiva tiene la ventaja de que no reduce significativamente de alternativas de inversión. Además, contra la opción negativa se puede decir que reduce la influencia del inversor sobre una empresa. Por ello, la inversión en empresas responsables y la no inversión en empresas irresponsables es más eficaz que la mera exclusión (Kolstad, 2016: 45-46).

Para poder llevar acabo cualquier acción que pueda considerarse como inversión responsable es indispensable contar con información fiable. Por ello es preciso contar con fuentes adecuadas, para lo cual existen ya guías de recursos para el inversor responsable que facilitan la gestión de la información ${ }^{6}$.

(b) Dentro de la segunda estrategia, el poder que el inversor tiene como accionista le sitúa en una posición de fuerza para impulsar la RC (Responsabilidad Corporativa) en la empresa. Entre las actuaciones que puede llevar a cabo se han señalado: la elección de los miembros del consejo de administración, la interacción con el equipo directivo, presentación y voto sobre cuestiones relacionadas con ESG, comunicación con los entes reguladores o la decisión de desinvertir (Olcese, Rodríguez y Alfaro, 2008: 225; Molthan, 2003: XIX-XX).

\footnotetext{
${ }^{6}$ Un ejemplo de estas guías es Broadhurst, et al. (2003).
} 
No es de extrañar que en los últimos años hayan proliferado las recomendaciones de la Unión Europea que animan a fomentar el compromiso de los accionistas, su participación en los asuntos de la sociedad, y a evitar que se vea la titularidad de acciones como una mera inversión que busca el retorno a corto plazo ${ }^{7}$.

\section{Iniciativas para el fomento de la inversión responsable}

\subsection{Introducción}

Son numerosas las iniciativas que han surgido desde distintas instancias para fomentar la inversión responsable. Se hará referencia, en primer lugar, a los índices bursátiles que permiten conocer cuáles son las empresas que tienen un mejor desempeño en responsabilidad y sostenibilidad dentro de un mercado de valores. En segundo lugar, se apuntarán algunas iniciativas internacionales de carácter voluntario en cuanto a su adhesión. Finalmente, se tratarán algunas iniciativas que combinan elementos voluntarios y obligatorios y que también pueden tener una incidencia relevante en el ámbito de la inversión responsable.

\subsection{Iniciativas en el ámbito bursátil}

La preocupación de los inversores por el buen destino de su inversión y por el comportamiento responsable de quienes reciben sus préstamos ha llevado a varias organizaciones de inversores y a otras instancias internacionales a elaborar índices cuyo cumplimiento deberán demostrar las empresas. Es, por lo tanto, una respuesta al interés de los inversores que quieren, por los motivos que sean, actuar de forma correcta y que pretenden evitar que sus inversiones terminen afectando negativamente a los derechos humanos 0 al medioambiente, o puedan verse implicadas en problemas de corrupción y soborno.

En este sentido cabe destacar algunos índices de sostenibilidad elaborados por el Grupo de Inversores Dow Jones. El DJSI World Index representa el 10\% de las empresas de distintos sectores basado en la "puntuación en sostenibilidad corporativa". Se evalúa a las empresas en función de unos principios de buenas prácticas en sostenibilidad corporativa. Da una importancia preeminente a la transparencia, al tiempo que anima a las empresas a ser evaluadas dentro de este proceso, en el que se asegura la confidencialidad de la información y que se basa en la confianza que se traduce en no acusar a quienes se retrasen (De Jonge, 2011: $55)$.

Las empresas se ven atraídas por aparecer en una lista de posibles destinatarios de inversión por estos fondos y ello les incentiva a cumplir con los criterios que establecen los índices. Los fondos de inversión juegan además un importante papel en la ejecución cuando demuestran su voluntad de suspender, cesar o reconsiderar su vinculación con empresas implicadas directa o indirectamente en actividades dañinas. Además de publicar sus políticas de inversión, los fondos también publican sus decisiones de dejar de invertir o de retirar de la lista a empresas. El efecto de estas medidas se extiende también a posibles decisiones que adopten otros inversores, grandes o pequeños, en todo el mundo (De Jonge, 2011: 56).

\footnotetext{
${ }^{7}$ En este sentido vid. la Propuesta de Directiva del Parlamento Europeo y del Consejo, por la que se modifica la Directiva 2007/36/CE en lo que respecta al fomento de la implicación a largo plazo de los accionistas y la Directiva 2013/34/UE en lo que respecta a determinados elementos de la declaración sobre gobernanza empresarial (Bruselas, 9.4.2014), COM(2014) 213 final 2014/0121 (COD).
} 
Además de los Dow Jones Sustainability Indexes y los FTSE4Good, que son los de mayor impacto en Europa, destaca el Domini 400 Social Index, para Estados Unidos (Olcese, Rodríguez y Alfaro, 2008: 228).

\subsection{Iniciativas internacionales}

\subsubsection{Introducción}

Para algunos autores, los compromisos asumidos a través de estas iniciativas, aunque comienzan siendo voluntarios, pasan después a ser de obligado cumplimiento, ya sea por los condicionantes legales, sociales o del mercado (Simons y Macklin, 2014: 84). Como ha señalado John Ruggie (2009), una multinacional que se jacte de cumplir con los deberes de diligencia debida solo puede demostrarlo cumpliendo de hecho con este compromiso. La interrelación que se genera entre gobiernos, empresas, sociedad civil, derecho privado, regulación estatal y autorregulación tanto a nivel nacional como internacional, unido a la presión jurídica, social, ética y del mercado hace difícil trazar una línea divisoria entre lo voluntario y lo jurídicamente vinculante (Simons y Macklin, 2014: 85; McBarnet, 2007: 54).

Sin embargo, muchos autores han puesto en tela de juicio la efectividad de la autorregulación a escala global y en especial en países menos desarrollados (Simons y Macklin, 2014: 85). Puede funcionar en países desarrollados, con instituciones fuertes, donde el estado incentiva a los actores privados a cumplir con los estándares sociales y medioambientales. Sin embargo esto no ocurre a nivel global, donde falta una entidad que mueva los hilos desde la sombra y oriente los comportamientos; falta lo que algunos autores denominan "shadow of hierarchy" (Simons y Macklin, 2014: 85).

Mientras no se rompa con el falso paradigma de que el objetivo de la empresa es maximizar los beneficios de los socios -cada vez más socios institucionales-, el cumplimiento de los códigos estará condicionado a su alineación con esa misión (Simons y Macklin, 2014: 88). Por eso las iniciativas voluntarias deben combinarse con normas vinculantes. En todo caso, las iniciativas basadas en la autorregulación pueden contribuir a crear un consenso sobre normas y crear unas expectativas de comportamiento, y en la medida en que pueden tener efectos en la reputación, las empresas pueden establecer procesos para demostrar su cumplimiento.

\subsubsection{Principios para la Inversión Responsable (PRI Initiative)}

La acción más importante de ámbito internacional son los Principios para la Inversión Responsable. Esta iniciativa nació amparada por dos instituciones de Naciones Unidas: la Iniciativa Financiera del PNUMA (Programa de las Naciones Unidas para el Medio Ambiente) y el Pacto Mundial de la ONU (UN Global Compact). Actualmente más de 1400 empresas del sector financiero (inversores institucionales) han firmado los Principios.

\footnotetext{
${ }^{8}$ Sobre estos tres índices, v. Olcese, Rodríguez y Alfaro (2008: 228-233). Estos autores destacan cuatro ventajas de la pertenencia a estos índices: a) permite una información comparativa con otras empresas en materia de RC; b) añade valor a la reputación y a la marca; c) facilitan una inversión sostenible; d) se difunde una imagen de la compañía implicada con su entorno.

${ }^{9}$ Es ilustrativo el ejemplo del caso Kasky v. Nike, traído por estos autores, en el que se empleó la normativa californiana de consumidores alegándose engaño por unas presuntas afirmaciones falsas sobre el cumplimiento por parte de la marca con sus códigos de conducta en fábricas en el extranjero. Nike Inc v. Kasky 123 S Ct 2554 (2003).
} 
Son 6 Principios que giran en torno al reconocimiento del carácter esencial de los asuntos medioambientales, sociales y de gobernanza (ESG) en las decisiones de inversión. De modo resumido, los principios son los siguientes:

- Analizar e incorporar ESG en la toma de decisiones de inversión: elección de empresas con buen comportamiento, descartar empresas no respetuosas, etc.

- Ser activos en las empresas en las que inviertan o participen, votando, haciendo propuestas al hilo de ESG, etc.

- Procurar que las empresas en las que invierten sean transparentes y publiquen su comportamiento en relación con ESG.

- Promover la aceptación e incorporación de los principios por otras empresas del sector de la inversión.

- Mejorar en la efectividad de la incorporación de los principios a través de la cooperación.

- Publicar las actuaciones y progresos propios en la puesta en práctica de los principios.

Los firmantes forman una red internacional de inversores que trabajan juntos para poner en práctica estos Principios. El objetivo de la iniciativa es comprender las implicaciones de la sostenibilidad para los inversores y apoyar a los firmantes a incorporar estos asuntos en la toma de decisiones de inversión y en sus prácticas de propiedad.

Como objetivo de fondo se pretende que, al incorporar los Principios, los firmantes contribuyan al desarrollo de un sistema financiero global más sostenible.

En cuanto a su funcionamiento, una característica de estos principios y de su aplicación es su carácter voluntario. Ofrecen un amplio abanico de acciones para incorporarlos. Una idea que inspira esta iniciativa es que el proceso de inversión responsable debe adaptarse a la estrategia, enfoque y recursos de cada entidad inversionista.

Al mismo tiempo esta iniciativa permite demostrar públicamente a los inversores su compromiso con la inversión responsable, así como colaborar y aprender con otras entidades sobre las implicaciones de los aspectos delicados a los que se refieren los principios para después incorporarlo en su praxis.

En definitiva, consiste en tener un conocimiento y una visión más amplia de los riesgos y oportunidades que tienen ante sí para colocar su capital de una forma que se alinee con los intereses a corto y largo plazo de sus clientes y beneficiarios.

Cuando menos, esta iniciativa está contribuyendo a reforzar y promover el paradigma de que las cuestiones relacionadas con el comportamiento medioambiental, social y de gobierno corporativo repercuten en el resultado financiero de las empresas, y ayuda a los inversores a ser conscientes de la importancia y responsabilidad de tomarse estos asuntos en serio, y cuando proceda, del deber que tiene de afrontarlos (Donald MacDonald citado en De Jonge, 2011: 56). 
4.3.3 Instituciones destinadas específicamente a orientar a las entidades financieras

Cabe citar como ejemplo la Corporación Financiera Internacional (IFC por sus siglas en inglés), perteneciente al Grupo Banco Mundial, que tiene como objetivo facilitar que empresas e instituciones financieras en países en desarrollo creen empleos, generen ingresos fiscales, mejoren el gobierno corporativo y el desempeño medioambiental y contribuyan a las comunidades locales (ICF, website).

4.3.4 El inversor como supervisor y garante del respeto del medioambiente y cuestiones sociales en la realización de grandes proyectos: los Principios de Ecuador

Los Principios de Ecuador son un marco de referencia adoptado por instituciones financieras para determinar, evaluar y afrontar los riesgos ambientales y sociales en los que se pueden al financiar ciertos proyectos (Equator Principles, website).

Comenzó como una iniciativa de 10 de los principales bancos de 7 países, en diálogo con las ONGs; ahora son 84 bancos, de 35 países, entre los que se encuentran el BBVA, Caixabank, Santander, Popular y Sabadell.

Como ha señalado algún autor, los Principios de Ecuador es una iniciativa que tiene como objetivo llevar a un número importante de entidades del sector financiero a exigir el respeto de un código de conducta que vinculará contractualmente a las empresas (De Jonge, 2011: 24).

Las políticas y principios del Grupo Banco Mundial y la Corporación Financiera Internacional (IFC) impulsan y orientan esta iniciativa. Su ámbito está limitado a instituciones que operan en la financiación grandes proyectos de infraestructuras e industriales. Las instituciones que forman parte de los Principios se comprometen a incorporarlos en sus políticas y decisiones de financiación y no financiarán proyectos en los que el cliente no vaya a cumplirlos.

El modo en que estos Principios operan es el siguiente. Como se ha dicho, las Entidades Financieras de los Principios de Ecuador (en adelante EPFI, por su denominación en inglés) se comprometen a no financiar grandes proyectos que no sean conformes con 10 principios específicos.

Se realiza una valoración de los proyectos según el proceso de evaluación medioambiental y social del IFC. Se les asigna una categoría en función del riesgo $(A, B \circ C)$. Si los proyectos se consideran de riesgo alto o medio (A O B), el solicitante del préstamo debe realizar una evaluación para hacer frente a los riesgos identificados. A continuación se lleva a cabo un proceso de consulta con los stakeholders locales afectados, y se les exigirá desarrollar un Sistema de Gestión Ambiental y Social y un Plan para cumplir con las normas aplicables. Si las normas no se cumplen, el cliente y el EPFI acordarán un Plan de Acción de los Principios de Ecuador que incluirá compromisos para cumplir con los requisitos. En este compromiso contractual radica la fortaleza de los Principios.

Además, las EPFI se comprometen a llevar a cabo actividades de asesoramiento. En concreto, explicarán a los clientes el contenido, la aplicación y las ventajas de aplicar los Principios al proyecto previsto y solicitarán al cliente que comunique a las EPFI su intención de adherirse a dichos Principios cuando posteriormente busquen financiación. 
Como se ha indicado anteriormente, es una de las iniciativas más próximas a gozar de una fuerza vinculante. No obstante, los Principios contienen una cláusula de "disclaimer" que deja claro que las EPFI consideran los Principios como una referencia no vinculante para el desarrollo de políticas, procedimientos y prácticas internas en cuestiones sociales y ambientales. No son vinculantes: ni crean derechos, ni generan responsabilidad sobre ninguna persona pública o privada. Sin embargo, como ya se ha señalado, la fortaleza de los principios radica en que en los documentos para la financiación se introducen pactos para asegurar que actuará de conformidad con los Principios. Si el prestatario no cumple con estos compromisos, las EPFI tratarán de facilitar que el prestatario cumpla en la medida de lo posible, y si no lo hace en el período de tiempo que se le conceda para ello, las EPFI se reservan el derecho de ejercer las acciones que estimen apropiadas.

El otro aspecto destacado en cuanto al control radica en que para los proyectos que entrañen más riesgo se requerirá el nombramiento de un experto independiente en materia medioambiental y/o social, o se requerirá al prestatario que cuente con expertos cualificados para que verifiquen la información de seguimiento que mantengan con las EPFI. Además, cada EPFI se compromete a publicar al menos anualmente informes sobre sus experiencias e implementación de los Principios teniendo en cuenta las exigencias de confidencialidad.

Siendo una iniciativa loable, existen ciertos aspectos de mejora, como una mayor transparencia en la información por parte de los bancos sobre su compromiso y respeto de los Principios (Banktrack I, 2016), un reconocimiento por parte de los EPFI de su responsabilidad de respetar los derechos humanos, así como la posibilidad de crear un mecanismo de reclamación en materia de derechos humanos al que puedan acudir las víctimas en proyectos financiados en el marco de los Principios (Banktrack II, 2016).

\subsection{Legislación europea y nacional}

Son también abundantes las iniciativas que parten de los Estados. Cabe señalar como ejemplo de esta modalidad la legislación inglesa que requiere a los fondos de pensiones declarar en qué medida han tenido en consideración los factores medioambientales, sociales o éticos a la hora de seleccionar, mantener o ejecutar sus inversiones (Molthan, 2003: XI).

La Unión Europea está fomentando este tipo de leyes a través de sus Directivas. Cabe destacar la propuesta de revisión de la Directiva sobre los derechos de los accionistas de $2014^{10}$, que busca incentivar que los inversores institucionales consideren mejor la información no financiera a la hora de tomar sus decisiones de inversión y que se impliquen más en las empresas en relación con estos asuntos.

Otra Directiva importante en este sentido es la 2014/95/UE, de 22 de octubre de 2014, sobre divulgación de información no financiera ${ }^{11}$. Para finales de 2016 los Estados miembros tendrán que transponer la Directiva y exigir a ciertas empresas, entre las que se encuentra las entidades de crédito, a incluir en su informe de gestión un estado no financiero que contenga información relativa a cuestiones

\footnotetext{
10 Informe de 12 de mayo de 2015, sobre la propuesta de Directiva del Parlamento Europeo y del Consejo por la que se modifica la Directiva 2007/36/CE en lo que respecta al fomento de la implicación a largo plazo de los accionistas y la Directiva 2013/34/UE en lo que respecta a determinados elementos de la declaración sobre gobernanza empresarial $(\operatorname{COM}(2014) 0213$ - C7-0147/2014 2014/0121(COD)).

${ }^{11}$ Directiva 2014/95/UE, de 22 de octubre de 2014, por la que se modifica la Directiva 2013/34/UE en lo que respecta a la divulgación de información no financiera e información sobre diversidad por parte de determinadas grandes empresas y determinados grupos.
} 
medioambientales y sociales, así como relativas al personal, al respeto de los derechos humanos y a la lucha contra la corrupción y el soborno. La Directiva quiere permitir gran flexibilidad en la actuación para tener en cuenta la "naturaleza multidimensional de la responsabilidad social de las empresas (RSE) y la diversidad de políticas de RSE aplicadas por las empresas, teniendo al mismo tiempo un nivel suficiente de comparabilidad que responda a las necesidades de los inversores y otras partes interesadas, así como la necesidad de ofrecer a los consumidores un acceso sencillo a la información sobre las repercusiones de las empresas en la sociedad" (considerando 3).

Esta Directiva debe suponer un espaldarazo a la inversión responsable en dos sentidos. Por un lado, por facilitar información al inversor sobre la realidad de las empresas en aspectos que van más allá de lo estrictamente financiero, y por otro, por exigir a las propias entidades de crédito (muchas veces inversores) a publicar cómo tienen en cuenta estas consideraciones.

Sin embargo, son muchos los que señalan que los términos que emplea la Directiva y las obligaciones que establece son excesivamente vagos, y que no se establecen criterios claros sobre cómo divulgar los riesgos e impactos negativos de las operaciones de la empresa (Chaplier y Gregor, 2014: 2) y los mecanismos de control de la veracidad de la información son en parte facultativos para los Estados. Existe, por tanto, el riesgo de que la Directiva se convierta en una herramienta de promoción para las empresas que gozan de cierto margen para publicar aquella información que más les beneficie.

También resulta problemático que las instituciones bancarias dependan de la información que las propias empresas-clientes les proporcionen (Simons y Macklin, 2014: 130).

5. Críticas a la inversión responsable y valoración final

Pese a que existen autores que ponen en duda la mayor rentabilidad de las inversiones responsables, sus argumentos son criticables. Señalan que es extremadamente complicado distinguir entre inversiones responsables e irresponsables debido a la falta de claridad y la incoherencia en algunos criterios empleados por los "fondos responsables". Ponen en duda incluso la falta de una definición clara de responsabilidad social corporativa (Vogel, 2016). Pero, si bien hay que reconocer que las incoherencias pueden llegar a ser inevitables, eso no puede llevarnos a negar la posibilidad de identificar y calificar un fondo como responsable.

Por otro lado, se pone en duda la existencia de información suficiente para llevar a cabo decisiones de inversión responsables. En primer lugar, porque son pocas las empresas que divulgan información sobre ESG. En segundo lugar, porque esa información es a menudo confusa e interesada (Vogel, 2016). Aunque estas afirmaciones son difíciles de negar, hay que contar con que cada vez son más las iniciativas encaminadas a garantizar una información más específica y que permita la comparación entre las empresas. No obstante, sí que es necesario generalizar sistemas de auditoría que garanticen la veracidad de las informaciones.

En todo caso, es indudable que el movimiento de la inversión responsable ha contribuido a concienciar a los inversores de que el riesgo que entraña toda inversión se reduce si se toman en cuenta factores como el medioambiente, los derechos humanos o el buen gobierno. 
La transparencia en la información, precisa y comprensible, que permita a los inversores, accionistas y consumidores conocer los datos relevantes en relación con el impacto de las empresas aspectos relacionados con ESG, es, sin lugar a dudas, la clave para que los intereses de los inversores y los de la economía global vayan en la misma dirección.

Bibliografía

BAN KI-MOON (2016), "Mensaje del Secretario General de la ONU”, Principios para la Inversión Responsable (Brochure en español), disponible en la siguiente dirección electrónica: https://www.unpri.org/about (fecha de consulta: 18 de julio de 2016).

BANKTRACK I (2016), The Equator Principles and transparency, disponible en la siguiente dirección electrónica: http://www.banktrack.org/show/pages/equator principles\#tab pages docume nts (fecha de consulta: 8 de septiembre de 2016).

BANKTRACK II (2016), The Equator Principles and human rights, disponible en la siguiente dirección electrónica: http://www.banktrack.org/show/pages/equator principles\#tab pages docume nts (fecha de consulta: 8 de septiembre de 2016).

BROADHURST, D., WATSON, J., MARSHALL, J., y MOLTHAN, P. (2003), Ethical and Socially Responsible Investment: A Reference Guide for Researchers, Walter de Gruyter, Múnich.

CHAPLIER, J. y GREGOR, F. (2014), ECCJ briefing. Assessment of the EU Directive on the disclosure of non-financial information by certain large companies, 2014.

COMISIÓN EUROPEA (2001), Libro Verde - Fomentar un marco europeo para la responsabilidad social de las empresas, COM (2001) 366 Final.

COMISIÓN EUROPEA (2002), Comunicación de la Comisión relativa a la responsabilidad social de las empresas: una contribución empresarial al desarrollo sostenible, COM/2002/0347 final.

COMISIÓN EUROPEA (2011), Estrategia renovada de la UE para 2011-2014 sobre la responsabilidad social de las empresas, COM (2011) 681 Final.

DE JONGE, A. (2011), Transnational Corporations and International Law. Accountability in the Global Business Environment, Elgar, Cheltenham.

IFC, Corporación Financiera Internacional. Grupo Banco Mundial, disponible en la siguiente dirección electrónica: http://www.ifc.org/ (fecha de consulta: el 18 de julio de 2016).

KOLSTAD, I. (2016), "Three questions about engagement and exclusion in responsible investment", Business Ethics: A European Review, vol. 25, Issue 1, pp. 45-58.

MCBARNET, D. (2007), "Corporate Social Responsability Beyond the Law, through Law, for Law: The New Corporate Accountability". En: MCBARNET, D., VOICULESCU, A. y CAMPBELL, T. (eds.), The New Corporate Accountability, Cambridge, Cambridge University Press, pp. 9-58.

MOLTHAN, P. (2003), "Introduction". En: BROADHURST, D., WATSON, J., MARSHALL, J., y MOLTHAN, P., Ethical and Socially Responsible Investment: A Reference Guide for Researchers, Múnich, Walter de Gruyter, pp XI-XXV.

OlCESE, A., RODRígueZ, M. A. y ALFARO, J. (2008), Manual de la empresa responsable y sostenible, McGraw-Hill, Madrid.

PRI, Principles for Responsible Investment website, disponible en la siguiente dirección electrónica: https://www.unpri.org/about/what-is-responsibleinvestment (fecha de consulta: el 18 de julio de 2016). 
RANSOME, W. y SAMPFORD, Ch. (2016), "Socially Responsible Investment: A Nascent Global Movement". En: RANSOME, W. y SAMPFORD, Ch., Ethics and Socially Responsible Investment: A Philosophical Approach, Farnham, Routledge, pp. 9-19.

RUGGIE, J. (2009), "Opening Satetement to UK Parliement Joint Committee on Human Rights", disponible en la siguiente dirección electrónica: http://business-humanrights.org/sites/default/files/media/bhr/files/Ruggiestatement-UK-3-Jun-2009.pdf (fecha de consulta: 18 de julio de 2016).

SIMONS, P. y MACKLIN, A. (2014), The Governance Gap. Extractive industries, human rights, and the home state advantage, Routledge, London-New York.

SPARKES, R. (2001), "Ethical Investment: Whose Ethics, which Investment?", Business Ethics: A European Review, vol. 10, pp. 194-205.

VOGEL, D. J., "Does Socially Responsible Investing Make Financial Sense?", The Wall Street Journal, 28 febrero 2016.

ZARBAFI, E. M. (2011), Responsible Investment and the Claim of Corporate Change. A Sensemaking Perspective on How Institutional Investors May Drive Corporate Social Responsibility, Gabler, Weisbaden.

ZERK, J. A. (2006), Multinationals and Corporate Social Responsibility, Cambridge University Press, Cambridge. 